\title{
INTEGRABILITY OF POWER SERIES
}

\author{
BABU RAM
}

\begin{abstract}
The object of this paper is to obtain generalization of certain results of Jain [3] and Woyczynski [5] concerning integrability of power series.
\end{abstract}

1. Introduction. A nondecreasing continuous real-valued function $\Phi$ defined on the nonnegative half line and vanishing only at the origin will be called an Orlicz function (OF). A function $\Phi \in \mathrm{OF}$ is said to satisfy $\Delta_{\alpha}(\alpha>0)$ condition for large $u$ if there are constants $c>0$ and $u_{0} \geqslant 0$ such that $\Phi(\alpha u) \leqslant c \Phi(u), u \geqslant u_{0}$. A convex Orlicz function $\Phi$ satisfying the conditions $\lim _{u \rightarrow 0}(\Phi(u) / u)=0$ and $\lim _{u \rightarrow \infty}(\Phi(u) / u)=\infty$ is called a Young function (YF). Function $\Phi$ belongs to YF if and only if it admits a representation $\Phi(u)=\int_{0}^{u} \phi(t) d t$, where $\phi(t), t \geqslant 0$, is positive, vanishing only at the origin, continuous on the right, nondecreasing and $\lim _{t \rightarrow \infty} \phi(t)=\infty$. Then $\Phi(u) / u \leqslant \phi(u)<\Phi(2 u) / u$. Let $\mathscr{M}$ be the class of Orlicz functions which satisfy the following condition:

$(\mathscr{M})$ There exists a convex function $\Lambda, \lambda>1$, and $0<\alpha<1$, such that the inequality $\Lambda(u) \leqslant \Phi^{\alpha}(u) \leqslant \lambda \Lambda(u)$ holds for all $u$.

Let $L_{\Phi}(X, \mu)$, where $\Phi \in \Delta_{\alpha}$, be the Orlicz space, that is, the set of all complexvalued measurable functions $f$ on a measure space $(X, \mu)$ such that the modular $\int_{X} \Phi(|f(x)|) d \mu$ is finite. By Hardy-Orlicz space $H_{\Phi}$ we mean a closed subspace of $L_{\Phi}(\langle 0,2 \pi\rangle, d x)$ spanned over trigonometric polynomials of the form $f(t)=$ $\sum_{n=0}^{N} a_{n} e^{i n t}$.

A sequence $\left\langle a_{n}\right\rangle$ of nonnegative numbers is said to be quasi monotone if for some $\alpha>0, a_{n+1} \leqslant a_{n}(1+\alpha / n)$ or equivalently $n^{-\beta} a_{n} \downarrow 0$ for some $\beta>0$.

Let $\Psi(x)$ be a nondecreasing positive function such that $\Psi(x) / x^{1-\delta}$ decreases as $x$ increases in $(0, a)$, where $a>0$ and $\delta$ is a small constant depending on $\Psi$. Then $\Psi^{\prime}(x)$ exists [2] almost everywhere and $\Psi^{\prime}(x) \geqslant 0$.

2. Results. We prove the following:

THEOREM. Let $\Phi \in \Delta_{\alpha} \cap \mathscr{M} \cap \mathrm{YF}$ and $f(x)=\sum_{k=0}^{\infty} a_{k} x^{k}, 0 \leqslant x<1$. If $\left\langle a_{n}\right\rangle$ is $a$ quasi monotone sequence such that

$$
\sum_{k=n}^{\infty}\left|a_{k}-a_{k+1}\right| \leqslant K a_{n},
$$

Received by the editors January $31,1984$.

1980 Mathematics Subject Classification. Primary 30A78; Secondary 42A32.

Key words and phrases. Orlicz function, $\Delta_{\alpha}$ condition, Young function, quasi-monotone sequence, Jensen's inequality. 
then the following four statements are equivalent:

$$
\begin{gathered}
\frac{\Phi(|f(x)|)}{\Psi(1-x)} \in L(0,1) ; \\
\frac{\Phi\left(\left|f\left(e^{i x}\right)\right|\right)}{\Psi(x)} \in L(0, \pi) ; \\
\sum_{n=1}^{\infty} \frac{\Phi\left(n a_{n}\right)}{n^{2} \Psi(1 / n)}<\infty ; \\
\sum_{n=1}^{\infty} \frac{\Phi\left(A_{n}\right)}{n^{2} \Psi(1 / n)}<\infty .
\end{gathered}
$$

In the special case of decreasing sequence $\left\langle a_{n}\right\rangle$ and $\Psi \equiv 1$ the above result was obtained by Woyczynski [5] and in the case $\Psi(x)=x^{-\gamma}, 0 \leqslant \gamma<1$, by Jain [3]. It may also be mentioned here that the author [4] also obtained some results of this type for an increasing, positive and convex function $\Phi$ with no restriction on $a_{n}$.

3. Lemmas. The proof of our theorem is based upon the following lemmas, which are generalizations of the lemmas of Woyczynski [5]. The proof of Lemma 1 also generalizes a classical Hardy's inequality (see [6, 9.16]). Let us write $F(x)=\int_{0}^{x} f(t) d t$.

LeMma 1. Let $\Phi \in \mathscr{M}, a \geqslant 0$. Then

$$
\int_{0}^{a} \frac{\Phi(F(x) / x)}{\Psi(x)} d x \leqslant K(\alpha) \int_{0}^{a} \frac{\Phi(f(x))}{\Psi(x)} d x
$$

Proof. By virtue of the assumption and of the Jensen's inequality there exists an $\alpha, 0<\alpha<1$, such that

$$
\Phi^{\alpha}(F(x) / x) \leqslant \lambda\left(\int_{0}^{x} \Phi^{\alpha}(f(t)) x^{-1} d t\right),
$$

whence

$$
\int_{0}^{a} \frac{\Phi(F(x) / x)}{\Psi(x)} d x \leqslant \lambda^{1 / \alpha} \int_{0}^{a} \frac{\left[\int_{0}^{x} \Phi^{\alpha}(f(t)) x^{-1} d t\right]^{1 / \alpha}}{\Psi(x)} d x .
$$

We consider the integral on the right-hand side of (3.1). We write

$$
H(x)=\int_{0}^{x} \Phi^{\alpha}(f(t)) d t .
$$

Then the integral under consideration becomes

$$
\int_{0}^{a} \frac{(H(x) / x)^{1 / \alpha}}{\Psi(x)} d x .
$$


Integration by parts yields

$$
\begin{aligned}
\int_{0}^{a} \frac{(H(x) / x)^{1 / \alpha}}{\Psi(x)} d x= & \frac{a(H(a) / a)^{1 / \alpha}}{\Psi(a)}-\int_{0}^{a} \frac{(H(x) / x)^{1 / \alpha-1} \Phi^{\alpha}(f(x))}{\alpha \Psi(x)} d x \\
& +\int_{0}^{a} \frac{(H(x) / x)^{1 / \alpha}}{\alpha \Psi(x)} d x+\int_{0}^{a} \frac{(H(x))^{1 / \alpha} \Psi^{\prime}(x)}{x^{1 / \alpha-1}(\Psi(x))^{2}} d x
\end{aligned}
$$

Thus, by the above, and by Hölder's inequality

$$
\begin{aligned}
\alpha\left(\frac{1}{\alpha}-1\right) \int_{0}^{a} \frac{(H(x) / x)^{1 / \alpha}}{\Psi(x)} d x & \leqslant \int_{0}^{a} \frac{(H(x) / x)^{1 / \alpha-1} \Phi^{\alpha}(f(x))}{\Psi(x)} d x \\
& \leqslant\left(\int_{0}^{a} \frac{\Phi(f(x))}{\Psi(x)} d x\right)^{\alpha}\left(\int_{0}^{a} \frac{(H(x))^{1 / \alpha} x^{-1 / \alpha}}{\Psi(x)} d x\right)^{1-\alpha} .
\end{aligned}
$$

Dividing both sides by the last factor on the right, we get

$$
(1-\alpha)\left(\int_{0}^{a} \frac{(H(x) / x)^{1 / \alpha}}{\Psi(x)} d x\right)^{\alpha} \leqslant\left(\int_{0}^{a} \frac{\Phi(f(x))}{\Psi(x)} d x\right)^{\alpha}
$$

whence

$$
\int_{0}^{a} \frac{(H(x) / x)^{1 / \alpha}}{\Psi(x)} d x \leqslant K(\alpha) \int_{0}^{a} \frac{\Phi(f(x))}{\Psi(x)} d x .
$$

The inequalities (3.1) and (3.3) then yield

$$
\int_{0}^{a} \frac{\Phi(F(x) / x)}{\Psi(x)} d x \leqslant K(\alpha) \int_{0}^{a} \frac{\Phi(f(x))}{\Psi(x)} d x .
$$

LEMMA 2. Let $\Phi \in \Delta_{\alpha} \cap$ YF and $a \geqslant 0$. If $f(x)$ is a nonnegative function, then

$$
\int_{0}^{a} \frac{\Phi(F(x))}{\Psi(x)} d x \leqslant K \int_{0}^{a} \frac{\Phi(x f(x))}{\Psi(x)} d x .
$$

Proof. Integration by parts and the given hypothesis yield

$$
\begin{aligned}
\int_{0}^{a} \frac{\Phi(F(x))}{\Psi(x)} d x & =\frac{a \Phi(F(a))}{\Psi(a)}-\int_{0}^{a} \frac{x \phi(F(x)) f(x)}{\Psi(x)} d x \\
& \geqslant-\int_{0}^{a} \frac{x f(x) \phi(F(x))}{\Psi(x)} d x .
\end{aligned}
$$

Now using Chen's inequality ([1], 3.10 for $t=1)$, we get on using $\Phi(u) / u \leqslant \phi(u)$ $<\Phi(2 u) / u$ and $\Phi(\alpha u) \leqslant c \Phi(u)$,

$$
\begin{aligned}
x f(x) \phi(F(x)) & =\operatorname{Max}\{x f(x) \phi(x f(x)), F(x) \phi(F(x))\} \\
& \leqslant\{\Phi(2 x f(x))+\Phi(2 F(x))\} \leqslant c[\Phi(x f(x))+\Phi(F(x))] .
\end{aligned}
$$


Therefore

$$
\int_{0}^{a} \frac{\Phi(F(x))}{\Psi(x)} d x \geqslant-c \int_{0}^{a} \frac{\Phi(x f(x))}{\Psi(x)} d x-c \int_{0}^{a} \frac{\Phi(F(x))}{\Psi(x)} d x
$$

whence

$$
\int_{0}^{a} \frac{\Phi(F(x))}{\Psi(x)} d x \leqslant K \int_{0}^{a} \frac{\Phi(x f(x))}{\Psi(x)} d x
$$

4. Proof of the Theorem. We shall prove the following implications:(2.1) $\Leftrightarrow(2.4)$; $(2.4) \Rightarrow(2.2) ;(2.2) \Rightarrow(2.3)$ and $(2.3) \Rightarrow(2.4)$.

$(2.1) \Rightarrow(2.4)$. In fact, if we write $1-x=y$, then we have for $1 /(n+1) \leqslant y \leqslant$ $1 / n, n \geqslant 2$,

$$
f(1-y) \geqslant \sum_{k=0}^{n} a_{k}(1-y)^{k} \geqslant(1-1 / n)^{n} \sum_{k=0}^{n} a_{k} \geqslant \frac{A_{n}}{4} .
$$

Thus

$$
f(1-y) \geqslant K A_{n} \quad \text { for } 1 /(n+1) \leqslant y \leqslant 1 / n \quad(n=2,3, \ldots) .
$$

Now

$$
\begin{aligned}
\sum_{n=1}^{\infty} \frac{\Phi\left(A_{n}\right)}{n^{2} \Psi(1 / n)} & \leqslant K \sum_{n=1}^{\infty} \int_{n}^{n+1} \frac{t^{-2} \Phi\left(A_{[t]}\right)}{\Psi(1 / t)} d t \\
& =K \sum_{n=1}^{\infty} \int_{1 / n+1)}^{1 / n} \frac{\Phi\left(A_{[1 / u]}\right)}{\Psi(u)} d u \\
& =K \int_{1 / 2}^{1} \frac{\Phi\left(A_{[1 / u]}\right)}{\Psi(u)} d u+K \sum_{n=2}^{\infty} \int_{1 /(n+1)}^{1 / n} \frac{\Phi\left(A_{[1 / u]}\right)}{\Psi(u)} d u \\
& \leqslant K+K \int_{0}^{1 / 2} \frac{\Phi(f(1-u))}{\Psi(u)} d u \\
& \leqslant K+K \int_{0}^{1} \frac{\Phi(f(x))}{\Psi(1-x)} d x<\infty
\end{aligned}
$$

(2.4) $\Rightarrow$ (2.1). We have

$$
\begin{aligned}
\int_{0}^{1} \frac{\Phi(f(x))}{\Psi(1-x)} d x & =\sum_{n=2}^{\infty} \int_{1-1 /(n+1)}^{1-1 / n} \frac{\Phi(f(x))}{\Psi(1-x)} d x \\
& =\sum_{n=2}^{\infty} \int_{1 / n}^{1 /(n-1)} \frac{\Phi(f(1-x))}{\Psi(x)} d x \\
& =\sum_{n=2}^{\infty} \int_{1 / n}^{1 /(n-1)} \frac{\Phi\left(\sum_{k=0}^{\infty} a_{k}(1-x)^{k}\right)}{\Psi(x)} d x
\end{aligned}
$$




$$
\begin{aligned}
& \leqslant \sum_{n=2}^{\infty} \int_{1 / n}^{1 /(n-1)} \frac{\Phi\left(\sum_{k=0}^{\infty} a_{k}(1-1 / n)^{k}\right)}{\Psi(x)} d x \\
& \leqslant \sum_{n=2}^{\infty} \frac{\Phi\left(\sum_{k=0}^{\infty} \sum_{j=n k}^{n(k+1)} a_{j}(1-1 / n)^{j}\right)}{n^{2} \Psi(1 / n)} \\
& \leqslant K \sum_{n=2}^{\infty} \frac{\Phi\left(\sum_{k=0}^{\infty} e^{-k} \sum_{j=0}^{n(k+1)} a_{j}\right)}{n^{2} \Psi(1 / n)} \\
& \leqslant K \sum_{n=2}^{\infty} \frac{\Phi\left(\sum_{k=0}^{\infty} e^{-k}(k+1) A_{n}\right)}{n^{2} \Psi(1 / n)} \\
& \leqslant K \sum_{n=2}^{\infty} \frac{\Phi\left(A_{n}\right)}{n^{2} \Psi(1 / n)}<\infty .
\end{aligned}
$$

(2.4) $\Rightarrow(2.2)$. We have

$$
\begin{aligned}
\left|\operatorname{Re} f\left(e^{i x}\right)\right| & =\left|\sum_{k=1}^{\infty} a_{k} \cos k x\right| \\
& =\left|\sum_{k=1}^{n} a_{k} \cos k x+\sum_{k=n+1}^{\infty} a_{k} \cos k x\right| \\
& =\left|\sum_{k=1}^{n} a_{k} \cos k x+\sum_{k=n}^{\infty}\left(a_{k}-a_{k+1}\right) D_{k}(x)-a_{n} D_{n}(x)\right| \\
& \leqslant A_{n}+O(1 / x) \sum_{k=n}^{\infty}\left|a_{k}-a_{k+1}\right|+O\left(x^{-1} a_{n}\right) .
\end{aligned}
$$

Then, on account of (2.0), it follows that

$$
\begin{aligned}
\int_{0}^{\pi} \frac{\Phi\left(\left|\operatorname{Re} f\left(e^{i x}\right)\right|\right)}{\Psi(x)} d x & =\sum_{n=1}^{\infty} \int_{\pi /(n+1)}^{\pi / n} \frac{\Phi\left(\left|\operatorname{Re} f\left(e^{i x}\right)\right|\right)}{\Psi(x)} d x \\
& =\sum_{n=1}^{\infty} \int_{\pi /(n+1)}^{\pi / n} \frac{\Phi\left[A_{n}+O(1 / x)\left(a_{n}\right)+O(1 / x)\left(a_{n}\right)\right]}{\Psi(x)} d x \\
& \leqslant K \sum_{n=1}^{\infty} \frac{\Phi\left(A_{n}+n a_{n}\right)}{n^{2} \Psi(1 / n)} .
\end{aligned}
$$

Since $\left\langle n^{-\beta} a_{n}\right\rangle$ is monotonically decreasing,

$$
A_{n}=\sum_{k=1}^{n} k^{-\beta} a_{k} k^{\beta} \geqslant n^{-\beta} a_{n} \sum_{k=1}^{n} k^{\beta} \geqslant K n^{-\beta} a_{n} n^{\beta+1}=K n a_{n} .
$$

Therefore, we have

$$
\int_{0}^{\pi} \frac{\Phi\left(\left|\operatorname{Re} f\left(e^{i x}\right)\right|\right)}{\Psi(x)} d x \leqslant K \sum_{n=1}^{\infty} \frac{\Phi\left(A_{n}\right)}{n^{2} \Psi(1 / n)}<\infty .
$$


It can be proved similarly that

$$
\int_{0}^{\pi} \frac{\Phi\left(\left|\operatorname{Im} f\left(e^{i x}\right)\right|\right)}{\Psi(x)} d x<\infty .
$$

$(2.2) \Rightarrow(2.3)$. Let $r(t)=\operatorname{Re} f\left(e^{i t}\right)$. The condition (2.0) implies that $\left\langle a_{n}\right\rangle$ is a sequence of bounded variation. Hence the Fourier series of $r$ converges for $x>0$. We write

$$
R(t)=\int_{0}^{t} r(x) d x \text { and } R_{1}(t)=\int_{0}^{t} R(x) d x .
$$

Integrating the Fourier series of $r(t)$, we have

$$
\begin{aligned}
R_{1}(t) & =\sum_{j=1}^{\infty} a_{j}(1-\cos j t) j^{-2} \\
& \geqslant \sum_{j=1}^{n} a_{j} j^{-2}(1-\cos j t) \\
& =2 \sum_{j=1}^{n} j^{-2} a_{j} \frac{\sin ^{2} j t}{2} \\
& \geqslant 2 \sum_{j=1}^{n} j^{-2} a_{j} \frac{4}{\pi^{2}} \frac{j^{2} t^{2}}{4}, \quad \pi /(n+1) \leqslant t \leqslant \pi / n \\
& \geqslant K t^{2} \sum_{j=1}^{n} a_{j} \geqslant K t^{2} n a_{n} \quad \text { due to the monotonicity of }\left\langle n^{-\beta} a_{n}\right\rangle .
\end{aligned}
$$

Now, by Lemma 1, we have

$$
\begin{aligned}
\sum_{n=1}^{\infty} \frac{\Phi\left(n a_{n}\right)}{n^{2} \Psi(1 / n)} & \leqslant \sum_{n=1}^{\infty} \int_{\pi /(n+1)}^{\pi / n} \frac{\Phi\left(x^{-2} R_{1}(x)\right)}{\Psi(x)} d x \\
& =\int_{0}^{\pi} \frac{\Phi\left(x^{-2} R_{1}(x)\right)}{\Psi(x)} d x \\
& \leqslant \int_{0}^{\pi} \frac{\Phi\left(x^{-2} \int_{0}^{x}|R(t)| d t\right)}{\Psi(x)} d x \\
& \leqslant \int_{0}^{\pi} \frac{\Phi\left(x^{-1} \int_{0}^{x} \frac{|R(t)|}{t} d t\right)}{\Psi(x)} d x \\
& \leqslant K \int_{0}^{\pi} \frac{\Phi\left(\frac{|R(x)|}{x}\right)}{\Psi(x)} d x \\
& \leqslant K \int_{0}^{\pi} \frac{\Phi(|r(x)|)}{\Psi(x)} d x \\
& \leqslant K \int_{0}^{\pi} \frac{\Phi\left(\left|f\left(e^{i x}\right)\right|\right)}{\Psi(x)} d x<\infty .
\end{aligned}
$$


$(2.3) \Rightarrow(2.4)$. Let $a(x)$ be a function equal to $a_{n}$ if $n-1 \leqslant x<n, n=1,2, \ldots$, and let $A(x)=\int_{0}^{x} a(t) d t$. The assumption

$$
\sum_{n=1}^{\infty} \frac{\Phi\left(n a_{n}\right)}{n^{2} \Psi(1 / n)}<\infty
$$

implies that $\Phi(\operatorname{ta}(t)) / \Psi(t)$ is integrable on the positive half line. Then, by virtue of Lemma 2, $\int_{0}^{a}(\Phi(A(t)) / \Psi(t)) d t<\infty$. But this is equivalent to the convergence of the series $\sum_{n=1}^{\infty} \Phi\left(A_{n}\right) / n^{2} \Psi(1 / n)$. This completes the proof of the theorem.

Acknowledgements. My thanks are due to Shri Subash Chander, Department of Mathematics, M. D. University, Rohtak for his kind encouragement during the preparation of this paper. Thanks are also due to the referee for his wise comments.

\section{REFERENCES}

1. Y. M. Chen, Some asymptotic properties of Fourier constants and integrability theorems, Math. Z. 68 (1957), 227-244.

2. Some further asymptotic properties of Fourier constants, Math. Z. 69 (1958), 105-120.

3. P. Jain, On the integrability of power series, Proc. Amer. Math. Soc. 42 (1974), 569-574.

4. B. Ram, Integrability theorems for power series, Colloq. Math. 31 (1974), 293-299.

5. W. A. Woyczynski, Positive coefficient elements of Hardy-Orlicz spaces, Colloq. Math. 21 (1970), 103-110.

6. A. Zygmund, Trigonometric series, Vol. I and II, Cambridge Univ. Press, 1968.

Department of Mathematics, M. D. University, RohtaK - 124001, INDia 